\section{Revista de CIENCIAS AMBIENTALES Tropical Journal of Environmental Sciences}

Revista de Ciencias Ambientales (Trop J Environ Sci) e-ISSN: 2215-3896

(Julio-Diciembre, 2021) . Vol 55(2): 141-156 DOI: https://doi.org/10.15359/rca.55-2.7

Open Access: www.revistas.una.ac.cr/ambientales e-mail: revista.ambientales@una.ac.cr Araiza-Ortiz M., Zambrano-González L., Mazari-Hiriart M. y Suzán G.

\title{
Evaluación de humedales someros tropicales semiáridos asociados al botulismo aviar
}

\author{
Evaluation of tropical semi-arid shallow wetlands associated to avian botulism
}

\author{
Marcela Areli Araiza-Ortiz ${ }^{1}$, Luis Zambrano-González², \\ Marisa Mazari-Hiriart ${ }^{3}$, Gerardo Suzán ${ }^{4}$
}

[Recibido: 22 de mayo 2020, Aceptado: 27 de abril 2021, Corregido: 15 de mayo 2021, Publicado: 1 de julio 2021]

\section{Resumen}

[Introducción]: Los humedales someros son relevantes para las aves acuáticas, actualmente son afectados por el cambio climático y las actividades humanas, esto se ha relacionado con brotes de botulismo aviar. [Objetivo]: Identificar cambios bióticos y fisicoquímicos en los humedales que podrían propiciar brotes de botulismo. Identificar fuentes de nutrientes que contribuyen a un estado eutrófico. [Metodología]: Evaluación de características bioticas (diversidad de zooplancton y macroinvertebrados) y fisicoquímicas (oxígeno disuelto, temperatura, solidos disueltos totales y transparencia) en 3 humedales (Trancas, Coyote y Silva) del centro de México. Identificación de la fuente de nutrientes mediante un análisis isotópico $\left(\delta^{13} \mathrm{C}\right.$ y $\left.\delta^{15} \mathrm{~N}\right)$ de los organismos acuáticos. [Resultados]: Después de la época de lluvias hubo una disminución en la diversidad de zooplancton e invertebrados, así como disminución del nivel y transparencia del agua en los sitios estudiados. Los valores de oxígeno disuelto, temperatura y sólidos disueltos totales fueron diferentes en los 3 sitios. Los valores de los isótopos estables de carbono $\left(\delta^{13} \mathrm{C}\right)$ y nitrógeno $\left(\delta^{15} \mathrm{~N}\right)$ de los organismos de Silva y Coyote, coinciden con valores de aguas negras, fertilizantes, maíz y trigo. [Conclusión]: Los humedales estudiados registraron cambios bióticos y fisicoquímicos a lo largo del periodo de estudio. En Silva y Coyote las fluctuaciones en el nivel del agua, las actividades humanas y la llegada de miles de aves acuáticas migratorias provocan una acumulación de materia orgánica en descomposición y nutrientes que vuelven a estos sitios eutróficos y propensos a tener brotes recurrentes de botulismo aviar.

Palabras claves: Aves acuáticas; isótopos estables; lagos someros eutróficos.

\section{Abstract}

[Introduction]: Shallow wetlands are relevant for waterfowl; they are currently affected by climatic changes and nutrients derived from agriculture. This has been associated with outbreaks of avian botulism. [Objective]: Identify changes in wetlands that can lead to botulism outbreaks, as well as identify the source of nutrients that may contribute to an eutrophic state. [Methodology]: Evaluation of biotic characteristics (diversity of zooplankton and macroinvertebrates) and physicochemical characteristics (dissolved oxygen, temperature, total dissolved solids and

1 Departamento de Etología, Fauna Silvestre y Animales de Laboratorio, Facultad de Medicina Veterinaria y Zootecnia, Posgrado en Ciencias Biológicas. Universidad Nacional Autónoma de México (UNAM), México; marcelaaraiza@hotmail.com, https://orcid. org/0000-0003-1915-4440

2 Laboratorio de Restauración Ecológica. Instituto de Biología, Universidad Nacional Autónoma de México (UNAM), México; zambrano@ ib.unam.mx, https://orcid.org/0000-0002-8632-0712

3 Laboratorio Nacional de Ciencias de la Sostenibilidad, Instituto de Ecología, Universidad Nacional Autónoma de México (UNAM), México; mazari@unam.mx, https://orcid.org/0000-0003-1646-2253

4 Departamento de Etología, Fauna Silvestre y Animales de Laboratorio, Facultad de Medicina Veterinaria y Zootecnia, Posgrado en Ciencias Biológicas, Universidad Nacional Autónoma de México (UNAM), México; gerardosuz@gmail.com, https://orcid.org/0000-0003-2508-6376

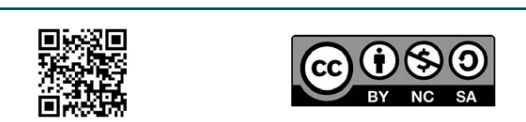
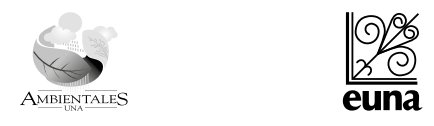


\section{Revista de CIENCIAS AMBIENTALES Tropical Journal of Environmental Sciences}

Revista de Ciencias Ambientales (Trop J Environ Sci) e-ISSN: 2215-3896

(Julio-Diciembre, 2021) . Vol 55(2): 141-156 DOI: https://doi.org/10.15359/rca.55-2.7

Open Access: www.revistas.una.ac.cr/ambientales e-mail: revista.ambientales@una.ac.cr Araiza-Ortiz M., Zambrano-González L., Mazari-Hiriart M. y Suzán G.

transparency) in 3 shallow wetlands (Silva, Coyote and Trancas) in central Mexico, as well as evaluation of values of stable isotopes of carbon $\left(\delta^{13} \mathrm{C}\right)$ and nitrogen $\left(\delta^{15} \mathrm{~N}\right)$ from organisms, in order to detect the input of nutrients to the aquatic ecosystem. [Results]: There was a decrease in zooplankton and invertebrate diversity as well as a decrease in water level and transparency at all 3 sites. The values of dissolved oxygen, temperature and total dissolved solids were different in the 3 sites. Values of stable carbon $\left(\delta^{13} \mathrm{C}\right)$ and nitrogen $\left(\delta^{15} \mathrm{~N}\right)$ isotopes from Silva and Coyote organisms coincide with values of stable isotopes found in sewage, fertilizers and grains such as corn and wheat. [Conclusion]: Wetlands in the study site registered biotic and physicochemical changes along the year. In Silva and Coyote water fluctuations, human activities and finally the arrive of thousands of migratory birds, produce an accumulation of dead organic matter and nutrients that made the system eutrophic and therefore more prone to present recurrent avian botulism outbreaks.

Keywords: Eutrophic shallow lakes; stable isotopes; waterfowl.

\section{Introducción}

Los humedales someros, ya sean naturales o artificiales, son relevantes para la reproducción y sobrevivencia de una diversidad de especies de aves acuáticas, tanto residentes como migratorias (Murakani et al., 2015). Sin embargo, este tipo de humedales son vulnerables al cambio climático asociado al calentamiento global, así como a la contaminación por actividades agropecuarias, principalmente en áreas tropicales y subtropicales (Menezes et al., 2019).

La variación interanual de las lluvias produce sequías y fluctuaciones en el nivel de agua que afectan al ecosistema acuático mediante el aumento de la eutroficación, de los niveles de salinización y anoxia, así como una alta producción de cianobacterias (Menezes et al., 2019). Además, la contaminación de los cuerpos de agua por agroquímicos es cada vez mayor conforme aumentan las necesidades de producción (Evans et al., 2019).

Los cambios provocados por la contaminación y las actividades agropecuarias afectan la biodiversidad de los humedales someros, incluyendo la composición de especies, la estructura trófica y el funcionamiento del ecosistema (Havens et al., 2016). Uno de los problemas que se ha asociado a estos cambios en los humedales someros son los brotes de botulismo aviar.

El botulismo es una enfermedad causada por la ingestión de la neurotoxina producida por la bacteria anaerobia Clostridium botulinum y es considerada la causa más importante de mortalidad en aves acuáticas (Rocke y Bollinger, 2007). La bacteria se considera ubicua y habita en el suelo, el polvo y la materia orgánica. La producción de la toxina ocurre durante la multiplicación de las esporas en condiciones ambientales favorables como: materia orgánica en descomposición, anoxia y temperaturas del agua mayores a $20^{\circ} \mathrm{C}$ (Babinszky et al., 2008).

Asimismo, los brotes se han asociado a fluctuaciones en el nivel del agua (Domínguez, 2009), a algunas actividades humanas que incrementan la carga de nutrientes (Ortiz y Smith, 1994), a la proliferación de cianobacterias (Murphy et al., 2000) y la presencia de peces del género Oreochromis spp (Nol et al., 2004), los cuales son introducidos muy comúnmente a este tipo de humedales.

\begin{tabular}{|c|c|c|c|c|}
\hline 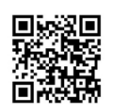 & (c) () () () & $\underset{\text { AMBEIETILES }}{8}$ & $\frac{1 \%}{2 \%}$ & 142 \\
\hline
\end{tabular}




\section{Revista de CIENCIAS AMBIENTALES Tropical Journal of Environmental Sciences}

Revista de Ciencias Ambientales (Trop J Environ Sci) e-ISSN: 2215-3896

(Julio-Diciembre, 2021) . Vol 55(2): 141-156 DOI: https://doi.org/10.15359/rca.55-2.7

Open Access: www.revistas.una.ac.cr/ambientales e-mail: revista.ambientales@una.ac.cr Araiza-Ortiz M., Zambrano-González L., Mazari-Hiriart M. y Suzán G.

En el estado de Guanajuato, en la parte central de México, se han presentado brotes de botulismo en varios humedales durante la temporada migratoria, cuando un gran número de aves se congrega. El brote más severo se presentó en la Presa de Silva durante el invierno de 1994-1995, en el cual murieron de 20000 a 40000 aves acuáticas (CEC, 1995). Un panel compuesto por especialistas de la Secretaría del Consejo de la Comisión para la Cooperación Ambiental (CCA) concluyó que la exposición a metales pesados y la contaminación del río Turbio, que alimentaba este embalse, causaron una mortalidad inicial en las aves. La presencia de cadáveres proporcionó un ambiente favorable para la reproducción de la bacteria causante del botulismo, por lo que la toxina se transfirió a otras aves cuando consumieron gusanos de moscas y otros invertebrados carroñeros (Rocke y Bollinger, 2007).

Después del brote, se cerró el afluente del río Turbio hacía la presa y se dragó el sedimento, desde entonces, el embalse se ha recargado solo con agua de lluvia y con un pequeño arroyo. Sin embargo, brotes de botulismo con mortalidad variable siguen ocurriendo en Silva y otros humedales someros casi todos los años. Registros de los últimos seis años indican que los brotes pueden comenzar desde noviembre y la mortalidad puede continuar hasta marzo. La cantidad de aves muertas varía de 50 a 900 individuos.

En Presa de Silva, una vez que comienza la mortalidad, se recolectan diariamente los cadáveres para disminuir la severidad del brote. Asimismo, se han realizado estudios patológicos en patos muertos para descartar una mortalidad inicial debido al cólera aviar (Pasteurella multocida), la cual es una enfermedad infecciosa que causa gran mortalidad en las aves acuáticas (Wray et al., 2016).

Debido al incremento de humedales someros que pueden presentar cambios en el nivel y calidad del agua debido al cambio climático, es necesario conocer las características bióticas y fisicoquímicas que podrían favorecer la presencia de la toxina botulínica y, por lo tanto, de brotes de botulismo aviar en las zonas tropicales o subtropicales. Esto permitiría planear algunas estrategias de manejo con el fin de reducir la frecuencia de los brotes y disminuir la mortalidad de las aves acuáticas.

El objetivo de este estudio es identificar cambios bióticos (diversidad de zooplancton y macroinvertebrados) y fisicoquímicos (transparencia del agua, temperatura, solidos disueltos totales, y oxígeno disuelto) en 2 humedales someros que presentan brotes frecuentes de botulismo aviar: Presa de Silva y Presa del Coyote; y en 1 humedal en donde no se han registrado brotes: Refugio de Trancas. Así como detectar la fuente de nutrientes que podrían favorecer la eutroficación en estos humedales.

\section{Metodología}

\subsection{Sitio de estudio}

El estudio se realizó en 3 humedales del estado de Guanajuato, ubicados dentro de la cuenca Lerma-Chapala en el centro de México, durante junio del 2015 a marzo del 2016 (Figura 1). Estos humedales son cruciales tanto para las aves residentes como migratorias, las cuales llegan por miles cada año. Los humedales en estudio están rodeados de zonas agrícolas y pequeños poblados (Figura 1).

\begin{tabular}{|c|c|c|}
\hline 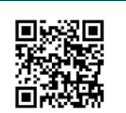 & (c) (i) (3) (2) & 143 \\
\hline
\end{tabular}




\section{Revista de CIENCIAS AMBIENTALES Tropical Journal of Environmental Sciences}

Revista de Ciencias Ambientales (Trop J Environ Sci) e-ISSN: 2215-3896

(Julio-Diciembre, 2021) . Vol 55(2): 141-156 DOI: https://doi.org/10.15359/rca.55-2.7

Open Access: www.revistas.una.ac.cr/ambientales e-mail: revista.ambientales@una.ac.cr Araiza-Ortiz M., Zambrano-González L., Mazari-Hiriart M. y Suzán G.

Presa de Silva y Presa del Coyote (Silva y Coyote de ahora en adelante) presentan brotes de botulismo frecuentes, son embalses no permanentes que se inundan en la época de lluvias, generalmente extendida de junio a septiembre, y permanecen secos de marzo a mayo. El tiempo de humedad o sequía es variable y depende de la cantidad de lluvia que se presente en el año. Refugio de Trancas (Trancas a partir de ahora) es un humedal permanente en donde no se han reportado brotes de botulismo.

Silva se encuentra en el municipio de San Francisco del Rincón, entre $20^{\circ} 57^{\prime} 31^{\prime \prime N}$ y $101^{\circ}$ 51' 38 "O, tiene una superficie aproximada de 49.8 hectáreas y una profundidad promedio de 1 metro. Se inunda cada año en la época de lluvias, el tiempo que se encuentra completamente seca depende de la cantidad de lluvia de cada año. Este humedal ha sido declarado sitio RAMSAR (Convención de Humedales de Importancia Internacional) por su importancia para un gran número de especies aviares.

Coyote está ubicado en el Municipio Cuerámaro, entre 20 38 '35” N y 101³6 '19” O, tiene una superficie de 204.47 hectáreas y una profundidad promedio de 1.5 metros. Al igual que Silva, se inunda en la época de lluvias por el río Turbio y los meses que dura seca dependen de la cantidad de lluvia de cada año. El agua se utiliza para el riego agrícola.

Trancas se encuentra en el municipio de Dolores Hidalgo, entre 2110'56” N y 101³'39” O, tiene una superficie de 11.34 hectáreas y una profundidad promedio de 1.5 metros. Es inundado por un sistema de canales y el agua se usa para riego agrícola.

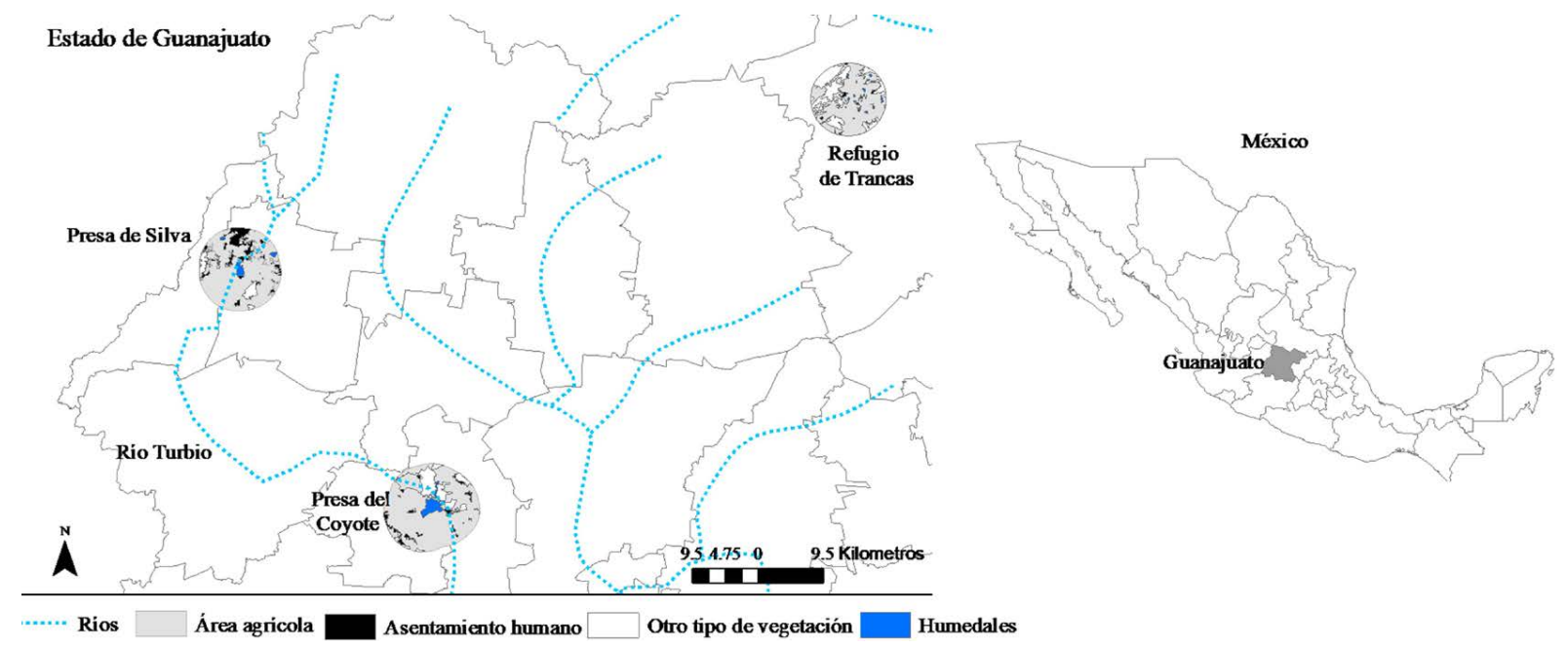

Figura 1. Ubicación de los humedales (Trancas, Silva y Coyote) del Estado de Guanajuato, México en los que se llevó a cabo el estudio descriptivo de algunas variables fisicoquímicas y bióticas.

Figure 1. Wetlands (Trancas, Silva and Coyote) of the state of Guanajuato, Mexico in which the descriptive study of some physicochemical and biotic variables was carried out.

\begin{tabular}{|c|c|c|c|c|c|}
\hline 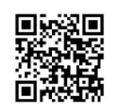 & 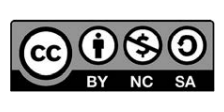 & $\underset{\text { AMBIENTALES }}{\leftrightarrow}$ & $\frac{1 \%}{2 \%}$ & 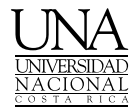 & 144 \\
\hline
\end{tabular}




\section{Revista de CIENCIAS AMBIENTALES Tropical Journal of Environmental Sciences}

Revista de Ciencias Ambientales (Trop J Environ Sci) e-ISSN: 2215-3896

(Julio-Diciembre, 2021) . Vol 55(2): 141-156 DOI: https://doi.org/10.15359/rca.55-2.7

Open Access: www.revistas.una.ac.cr/ambientales e-mail: revista.ambientales@una.ac.cr Araiza-Ortiz M., Zambrano-González L., Mazari-Hiriart M. y Suzán G.

\subsection{Muestreo y procesamiento de muestras}

\subsubsection{Características bióticas}

Entre agosto del 2015 (época no migratoria) y enero del 2016 (época migratoria), se colectó zooplancton y macroinvertebrados acuáticos en puntos elegidos al azar. Se reunió el zooplancton utilizando una red de $60 \mu \mathrm{m}$, mientras que para la colecta de macroinvertebrados se utilizó una red de $2 \mathrm{~mm}$. Posteriormente, en el laboratorio se contó e identificó los individuos existentes en la muestra. Para el zooplancton se observó la cantidad existente en una muestra de 0.05 $\mathrm{ml}$. Ambos grupos se contaron e identificaron usando un microscopio estereoscópico $(4 \mathrm{x})$.

\subsubsection{Características fisicoquímicas}

En cada humedal se eligió puntos al azar para evaluar características fisicoquímicas como: transparencia del agua, solidos disueltos totales (SDT), temperatura (T) y oxígeno disuelto (OD). Durante junio del 2015 y enero del 2016 se midió la transparencia del agua utilizando un disco de Secchi, el cual mide la transparencia según la profundidad a la que desaparece el disco del alcance visual. Para poder evaluar los cambios entre los meses, se midió la profundidad total en el mismo punto donde se tomó la medida del disco de Secchi y posteriormente se obtuvo el porcentaje de la distancia a la que se podía ver el disco con respecto a la profundidad del sitio de muestreo (PMS-PT).

En enero del 2016, se evaluó la cantidad de SDT, la T y el OD mediante una sonda multiparamétrica especializada (YSI 6600; YSI Inc., Yellow Springs, OH, U.S.A). En Coyote, se evaluó únicamente los SDT y la temperatura ya que el nivel del agua era muy bajo para que el equipo permitiera medir el OD.

\subsubsection{Identificación de la fuente de nutrientes}

Para identificar la fuente de nutrientes hacia los humedales, de septiembre del 2015 a marzo del 2016 se recolectó semillas, zooplancton, macroinvertebrados y muestras de patos (sangre e hígado), y se obtuvo el valor de los isótopos estables de carbono $\left(\delta^{13} \mathrm{C}\right)$ y nitrógeno $\left(\delta^{15} \mathrm{~N}\right)$. Se ha demostrado que los valores de $\delta^{15} \mathrm{~N}$ de los organismos acuáticos reflejan de manera confiable el aporte de nitrógeno de la tierra al agua, esto se ha detectado tanto en productores y consumidores primarios (McClelland y Valiela, 1998), así como en las plumas de las aves acuáticas (Hebert y Wassenaar, 2001). Los valores de $\delta^{13} \mathrm{C}$ también son un indicador confiable de la fuente de materia orgánica para los ecosistemas acuáticos (Thornton y McManus, 1994).

Para obtener las muestras de sangre de los patos, se realizó capturas con el uso de redes de niebla con el permiso científico SEMARNAT-FAUT-0250. Las redes fueron colocadas antes del anochecer y permanecieron abiertas durante toda la noche. Posterior al muestreo, se liberaron los animales en el sitio de captura. Se obtuvieron las muestras de hígado de patos donados

\begin{tabular}{|c|c|c|}
\hline 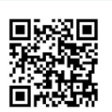 & (c) $(9)(9)$ & 145 \\
\hline
\end{tabular}




\section{Revista de CIENCIAS AMBIENTALES Tropical Journal of Environmental Sciences}

Revista de Ciencias Ambientales (Trop J Environ Sci) e-ISSN: 2215-3896

(Julio-Diciembre, 2021) . Vol 55(2): 141-156 DOI: https://doi.org/10.15359/rca.55-2.7

Open Access: www.revistas.una.ac.cr/ambientales e-mail: revista.ambientales@una.ac.cr Araiza-Ortiz M., Zambrano-González L., Mazari-Hiriart M. y Suzán G.

legalmente por cazadores. La colecta de plancton y macroinvertebrados se encuentra descrita en la sección de variables bióticas.

Se mantuvieron las muestras en congelación hasta su procesamiento en el laboratorio, el cual consistió en el secado a $60{ }^{\circ} \mathrm{C}$ por 72 horas, su posterior pulverización con ayuda de un mortero y su colocación en cápsulas de estaño especiales para su envío al Laboratorio de Isótopos de la Universidad de Davis, California.

Para determinar las firmas isotópicas se utiliza la espectrometría de masas, donde la proporción de isótopos obtenida en la muestra se compara con los estándares internacionales, es decir, la formación Beelemita Pee Dee de Carolina del Sur (PDB) para el carbono (Craig, 1957) y el nitrógeno atmosférico $\left(\mathrm{N}_{2}\right)$ para el nitrógeno (Jardine et al., 2003). El resultado se obtiene mediante la Ecuación 1 (Michener y Lajtha, 2007).

$$
\delta \% \mathrm{X}=\left\{\left[\mathrm{R}_{\text {muestra }}-\mathrm{R}_{\text {estándar }}\right] / \mathrm{R}_{\text {estándar }}\right\}^{*} 1000(\text { E.1) }
$$

Donde:

$\mathrm{X}$ es la diferencia de la muestra con el estándar expresado en partes por mil (\%o).

$\mathrm{R}_{\text {muestra }}$ es la proporción de isótopos pesados y ligeros para la muestra.

$\mathrm{R}_{\text {estándar }}$ es la proporción de isotopos pesados y ligeros para el estándar.

\subsection{Análisis estadístico}

Para evaluar la diversidad de zooplancton y macroinvertebrados en los diferentes sitios y en la época migratoria y no migratoria, se calculó el índice de Shanon con el software EstimateS, luego se realizó la comparación del índice de Shanon entre sitios y entre la época migratoria y no migratoria, haciendo uso de la prueba t de Hutcheson específica para comparar el índice de Shannon.

Para comparar los valores de las variables fisicoquímicas y de los isótopos estables entre sitios, se utilizó el análisis no paramétrico de Kruskall-Wallis, debido a que los datos recabados no cumplían con el supuesto de homogeneidad de varianza. Se comparó sitio por sitio mediante la prueba de comparación de medianas para dos muestras independientes. En el caso del OD, se hizo uso de la prueba de U de Mann-Whitney para comparar dos medias.

Utilizando el Software ArcMap 10.1 y un mapa digital de uso de la tierra y vegetación (escala 1: 250 000, https://www.inegi.org.mx/temas/usosuelo/default.html\#Descargas), se generó un área de 5 kilómetros alrededor de cada embalse y calculó el porcentaje del área ocupada con agricultura. Posteriormente, haciendo uso del software estadístico SPSS, se realizó la prueba de correlación de Spearman para evaluar la correlación de los valores de los isótopos estables de $\delta \mathrm{N}^{15} \mathrm{y}$ de $\delta \mathrm{C}^{13}$ en los organismos con el porcentaje de agricultura que rodea los humedales.

\section{Resultados}

El estudio comenzó en junio del 2015 cuando, Coyote y Silva se inundaron, y finalizó en marzo del 2016, cuando había bajos niveles de agua.

\begin{tabular}{|c|c|c|c|c|}
\hline 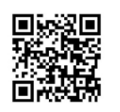 & (c) (i) (\$) & $\underset{\text { AMBENETILES }}{3}$ & $\frac{1 \%}{2 \%}$ & 146 \\
\hline
\end{tabular}




\section{Revista de CIENCIAS AMBIENTALES Tropical Journal of Environmental Sciences}

Revista de Ciencias Ambientales (Trop J Environ Sci) e-ISSN: 2215-3896

(Julio-Diciembre, 2021) . Vol 55(2): 141-156 DOI: https://doi.org/10.15359/rca.55-2.7

Open Access: www.revistas.una.ac.cr/ambientales e-mail: revista.ambientales@una.ac.cr Araiza-Ortiz M., Zambrano-González L., Mazari-Hiriart M. y Suzán G.

\subsection{Características bióticas}

Para evaluar la diversidad de zooplancton y macroinvertebrados, se colectaron organismos pertenecientes a 2 Filos, 2 Clases, 2 Subclases, 4 Ordenes, 1 Suborden y 3 familias (Cuadro 1). En el mes de agosto, la diversidad de zooplancton y macroinvertebrados fue diferente en los 3 sitios. Trancas presentó una mayor diversidad que los otros sitios, mientras que en Coyote se registró la menor diversidad. Una vez terminó la temporada de lluvias, el nivel de agua disminuyó constantemente y en enero la diversidad de zooplancton y macroinvertebrados disminuyó considerablemente en Trancas y Silva. En Coyote, la diversidad de ambos grupos no tuvo un cambio significativo de agosto a enero (Cuadro 1).

Cuadro 1. Diversidad de zooplancton y macroinvertebrados durante la época no migratoria (agosto del 2015) y migratoria (enero del 2016) de aves acuáticas, en 3 humedales someros, Refugio de Trancas, Presa del Coyote y Presa de Silva, del Estado de Guanajuato, México.

Table 1. Diversity of zooplankton and macroinvertebrates during non-migratory (August 2015) and migratory (January 2016) season of waterfowl in three shallow wetlands, Refugio de Trancas, Presa del Coyote y Presa de Silva, in the state of Guanajuato, Mexico.

\begin{tabular}{|c|c|c|c|c|c|c|}
\hline & \multicolumn{6}{|c|}{ Diversidad de zooplancton y macroinvertebrados } \\
\hline & \multicolumn{3}{|c|}{ Antes de la época migratoria } & \multicolumn{3}{|c|}{ Después de la época migratoria } \\
\hline & Trancas $^{*}$ & Coyote & Silva & Trancas & Coyote & Silva \\
\hline \multicolumn{7}{|l|}{ Zooplancton } \\
\hline Filo Rotifera & $4(15.3)$ & $2(133)$ & $6(41.8)$ & $3(39.3)$ & $3(0.7)$ & $5(12.5)$ \\
\hline Filo Tardigrada & $4(0.3)$ & $2(0)$ & $6(0)$ & $3(0)$ & $3(0)$ & $5(0)$ \\
\hline Subclase Copepoda & $4(6.8)$ & $2(2.5)$ & $6(18)$ & $3(3)$ & $3(2.7)$ & $5(2.5)$ \\
\hline Suborden Cladocera & $4(4.3)$ & $2(45.5)$ & $6(1.5)$ & $3(1)$ & $3(1)$ & $5(0.5)$ \\
\hline \multicolumn{7}{|l|}{ Macroinvertebrados } \\
\hline Clase Ostracoda & $2(0)$ & $2(0)$ & $3(2)$ & $2(0)$ & $2(0)$ & $5(0)$ \\
\hline Clase Gastropoda & $2(0)$ & $2(0)$ & $3(0.3)$ & $2(0)$ & $2(0)$ & $5(0)$ \\
\hline Subclase Hirudinea & $2(0.5)$ & $2(0)$ & $3(0)$ & $2(0)$ & $2(0)$ & $5(0)$ \\
\hline Orden Diptera & $2(1)$ & $2(0)$ & $3(0)$ & $2(0)$ & $2(0)$ & $5(0)$ \\
\hline Orden Coleoptera & $2(0)$ & $2(1)$ & $3(0)$ & $2(0)$ & $2(0)$ & $5(0)$ \\
\hline Orden Ephemeroptera & $2(1.5)$ & $2(0)$ & $3(1.3)$ & $2(0.5)$ & $2(0)$ & $5(0)$ \\
\hline Orden Odonata & $2(3.5)$ & $2(0)$ & $3(2.7)$ & $2(2.5)$ & $2(0)$ & $5(0)$ \\
\hline Familia Chironomidae & $2(0)$ & $2(20)$ & $3(2)$ & $2(0.5)$ & $2(3)$ & $5(0)$ \\
\hline Familia Sialidae & $2(0)$ & $2(0.5)$ & $3(0)$ & $2(0)$ & $2(0)$ & $5(0)$ \\
\hline Familia Corixidae & $2(2.5)$ & $2(10)$ & $3(3.2)$ & $2(2.5)$ & $2(0.5)$ & $5(0)$ \\
\hline Índice de Shannon & 1.74 & 0.98 & 1.4 & 0.94 & 1.05 & 0.46 \\
\hline Varianza para el índice de Shannon $\left(\mathrm{S}^{2} \mathrm{H}\right)$ & 0.0030 & 0.0055 & 0.0117 & 0.0312 & 0.0232 & 0.0345 \\
\hline Hutcheson t-test & \multicolumn{3}{|c|}{ Antes de la época migratoria } & \multicolumn{3}{|c|}{ Después de la época migratoria } \\
\hline Trancas-Coyote & \multicolumn{3}{|c|}{$\mathrm{t}=8.24, \mathrm{p}<0.05$} & \multicolumn{3}{|c|}{$\mathrm{t}=2.49, \mathrm{p}<0.05$} \\
\hline Trancas-Silva & \multicolumn{3}{|c|}{$\mathrm{t}=2.81, \mathrm{p}<0.05$} & \multicolumn{3}{|c|}{$\mathrm{t}=0.04, \mathrm{p}=0.97$} \\
\hline Silva-Coyote & \multicolumn{3}{|c|}{$\mathrm{t}=3.2, \mathrm{p}<0.05$} & \multicolumn{3}{|c|}{$\mathrm{t}=3.91, \mathrm{p}<0.05$} \\
\hline Trancas & \multicolumn{6}{|c|}{$\mathrm{t}=4.33, \mathrm{p}=0.000006$} \\
\hline Coyote & \multicolumn{6}{|c|}{$\mathrm{t}=0.413, \mathrm{p}=0.688$} \\
\hline Silva & \multicolumn{6}{|c|}{$\mathrm{t}=4.37, \mathrm{p}=0.00009$} \\
\hline
\end{tabular}

* Número de muestra (media)

(cc)




\section{Revista de CIENCIAS AMBIENTALES Tropical Journal of Environmental Sciences}

Revista de Ciencias Ambientales (Trop J Environ Sci) e-ISSN: 2215-3896

(Julio-Diciembre, 2021) . Vol 55(2): 141-156 DOI: https://doi.org/10.15359/rca.55-2.7

Open Access: www.revistas.una.ac.cr/ambientales e-mail: revista.ambientales@una.ac.cr Araiza-Ortiz M., Zambrano-González L., Mazari-Hiriart M. y Suzán G.

\subsection{Características fisicoquímicas}

En el mes de junio, Silva y Trancas presentaron agua clara (PMS-PT $=87 \%$ para ambos sitios), mientras que Coyote ya presentaba agua turbia (PMS-PT = $52.8 \%$ ). Se observó la presencia de macrófitos en los 3 sitios. En enero, cuando miles de aves migratorias se encontraban en los embalses, la transparencia del agua de Silva había disminuido considerablemente (PMS-PT $=32 \%)$ con respecto a Trancas (PMS-PT $=75 \%$ ) y Coyote (PMS-PT $=50 \%)$. En ese momento, la mayoría de los macrófitos de Silva había muerto, el promedio de OD estaba muy bajo y se registraron zonas completamente anóxicas, además de temperaturas superiores a $20{ }^{\circ} \mathrm{C}$, las cuales fueron significativamente más altas que en Trancas. Los valores de SDT en Coyote y Silva también fueron significativamente más altos que en Trancas (Cuadro 2).

Cuadro 2. Características fisicoquímicas medidas durante enero del 2016 en 3 humedales someros, Refugio de Trancas, Presa de Silva y Presa del Coyote en el Estado de Guanajuato, México.

Table 2. Physicochemical characteristics measured during January 2016 in three shallow wetlands, Refugio de Trancas, Presa de Silva y Presa del Coyote in the state of Guanajuato, Mexico.

\begin{tabular}{llll}
\hline & \multicolumn{1}{c}{ Sólidos disueltos totales $\mathbf{~ m g} / \mathbf{L}^{*}$} & \multicolumn{1}{c}{ Temperatura ${ }^{\circ} \mathbf{C}$} & \multicolumn{1}{c}{ Oxígeno disuelto $\mathbf{~ m g} / \mathbf{L}^{-}$} \\
\hline Trancas & $7(34.7) 5.03$ & $7(14.5) 0.5$ & $7(4.3) 1.2$ \\
Silva & $6(255) 16.1$ & $6(22.5) 0.1$ & $6(0.07) 0.1$ \\
Coyote & $3(966.3) 21.6$ & $3(19.7) 1.6$ & \\
Prueba de Kruskal-Wallis & $X^{2}(2)=9.38, \mathrm{p}=0.009$ & $X^{2}(2)=11.3, \mathrm{p}=0.003$ & Prueba de U Mann-Whitney \\
& & & $\mathrm{U}=0.000, \mathrm{p}=0.004$ \\
Prueba de comparación de medianas & $\mathrm{p}=0.033$ & \\
Trancas-Coyote & $\mathrm{p}=0.002$ & $\mathrm{p}=0.001$ & \\
Trancas-Silva & $\mathrm{p}=0.077$ & $\mathrm{p}=0.656$ & \\
Silva-Coyote & $\mathrm{p}=0.077$ & & \\
\hline
\end{tabular}

*Número de muestra (media) desviación estándar

\subsection{Identificación de la fuente de nutrientes}

Para la identificación de la fuente de nutrientes muestreamos 27 patos, 3 capturados mediante red de niebla, de los cuales extrajimos muestras de sangre y 24 donados por cazadores, de los cuales obtuvimos muestras de hígado. Así mismo, obtuvimos muestras de dos familias de macroinvertebrados y semillas de dos especies (Parthenium spp. en Silva y Polygonum spp. en Trancas). También colectamos huevos de insecto (no identificados) y plancton (Cuadro 3).

Los valores de los isótopos $\delta^{15} \mathrm{~N}$ y $\delta^{13} \mathrm{C}$ de los organismos fueron diferentes en los 3 humedales muestreados, sin embargo, tuvieron una correlación positiva con el porcentaje ocupado por agricultura dentro del área de $5 \mathrm{~km}$ alrededor de cada humedal (Cuadro 3). Los valores de $\delta^{15} \mathrm{~N}$ de las semillas y el plancton de Silva y Coyote fueron más altos que los de Trancas, de $13.4 \%$ a $23.5 \%$. Al igual que los valores de $\delta^{13} \mathrm{C}$ de organismos de Presa del Coyote (de $-13 \%$ a $-19.3 \%$ ) (Cuadro 3).

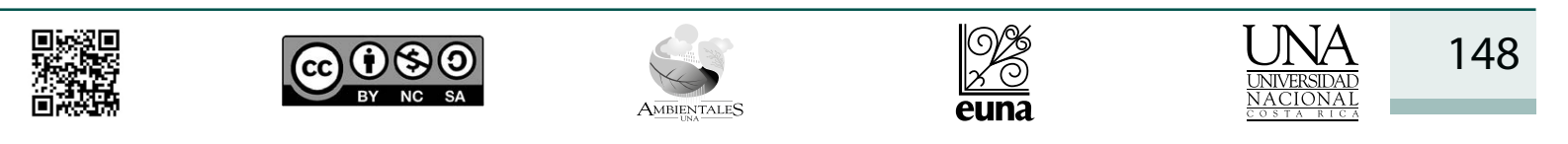




\section{Revista de CIENCIAS AMBIENTALES Tropical Journal of Environmental Sciences}

Revista de Ciencias Ambientales (Trop J Environ Sci) e-ISSN: 2215-3896

(Julio-Diciembre, 2021) . Vol 55(2): 141-156 DOI: https://doi.org/10.15359/rca.55-2.7

Open Access: www.revistas.una.ac.cr/ambientales e-mail: revista.ambientales@una.ac.cr Araiza-Ortiz M., Zambrano-González L., Mazari-Hiriart M. y Suzán G.

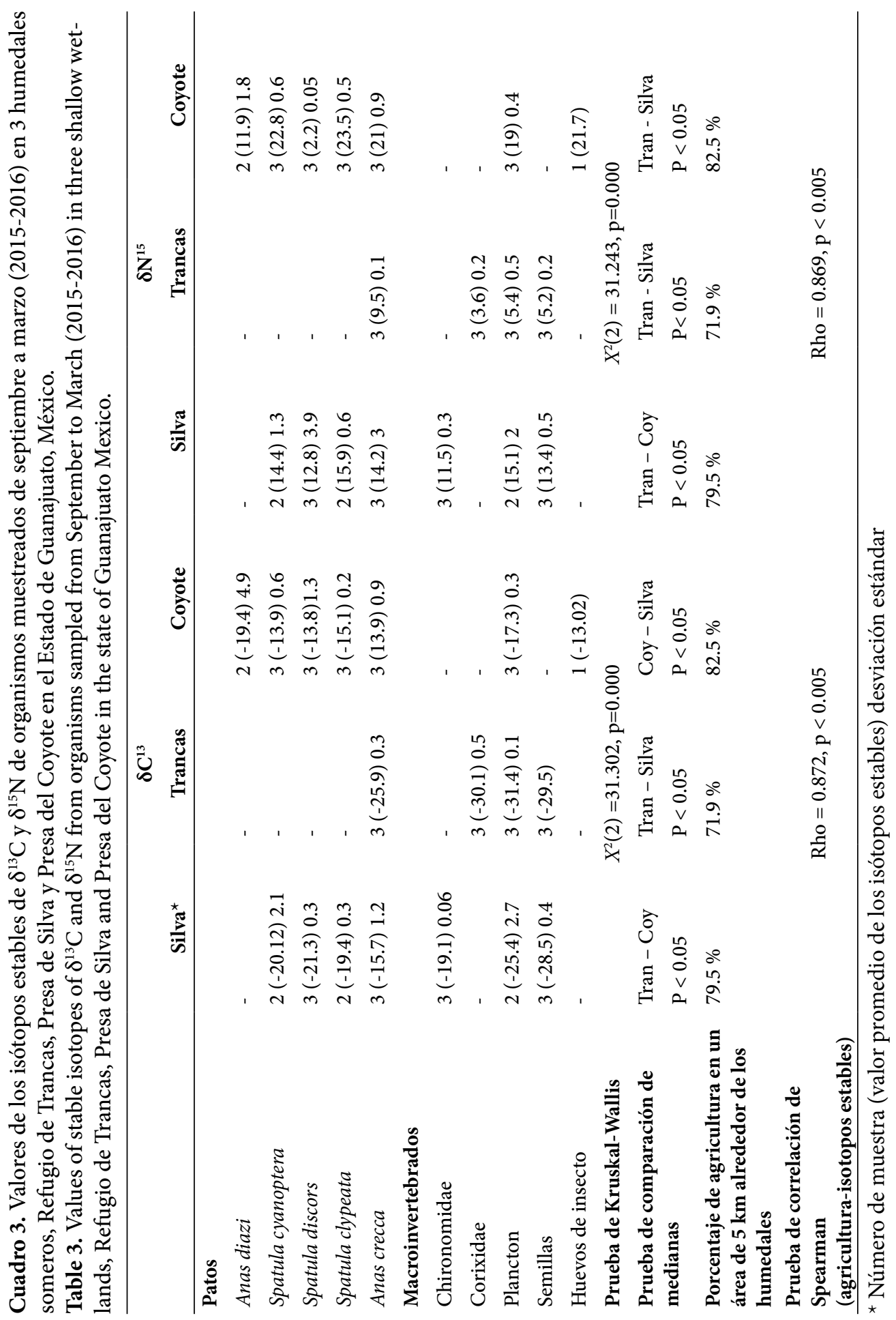




\section{Revista de CIENCIAS AMBIENTALES Tropical Journal of Environmental Sciences}

Revista de Ciencias Ambientales (Trop J Environ Sci) e-ISSN: 2215-3896

(Julio-Diciembre, 2021) . Vol 55(2): 141-156 DOI: https://doi.org/10.15359/rca.55-2.7

Open Access: www.revistas.una.ac.cr/ambientales e-mail: revista.ambientales@una.ac.cr Araiza-Ortiz M., Zambrano-González L., Mazari-Hiriart M. y Suzán G.

\section{Discusión}

El objetivo de este estudio fue identificar cambios bióticos y fisicoquímicos en humedales someros que han presentado brotes de botulismo aviar y en un humedal donde no se han registrado brotes, así como detectar la fuente de nutrientes hacia estos embalses. Los 3 humedales evaluados sufrieron cambios de junio del 2015 a marzo del 2016; sin embargo, los cambios más importantes se observaron en Silva, sitio donde los brotes de botulismo son frecuentes. En este humedal se notó un cambio importante de agua clara a turbia de junio a marzo, así como una disminución en la diversidad de macroinvertebrados y de zooplancton.

Cada año, al inundarse Silva tras varios meses de completa sequía, los pobladores locales introducen peces del género Oreochromis spp juveniles, con el fin de pescarlos cuando alcancen la edad adulta. La introducción de peces a los humedales someros se ha asociado con una disminución en la transparencia del agua ya que proporcionan materia orgánica a través de sus heces y, por sus hábitos alimenticios, aumentan la carga de nutrientes a la columna de agua mediante la resuspensión del sedimento (Comín y Herrera, 2000; Araújo et al., 2015).

Además del material orgánico aportado por los peces, la muerte de vegetación terrestre al inundarse Silva añade grandes cantidades de materia orgánica en descomposición. En el mes de septiembre, miles de aves acuáticas migratorias llegan a los embalses, proporcionando aún más nutrientes a través de sus heces (Comín y Herrera, 2000). También la herbivoría por parte de las aves acuáticas puede disminuir la biomasa de los macrófitos, de forma que favorece el cambio de un estado claro a un estado turbio (van Altena et al., 2016). Estos cambios se han asociado a una disminución de la diversidad tanto de zooplancton como de macroinvertebrados (Usio et al., 2017).

La gran cantidad de nutrientes causada por la introducción de los peces y la llegada de aves migratorias, además de la materia orgánica muerta producto de la descomposición de la vegetación terrestre, pueden ser la causa de eutrofización y, por lo tanto, de la anoxia encontrada en el sedimento de Silva (Scheffer y Ness, 2007).

Los cambios observados en la presa del Coyote no fueron tan marcados, sin embargo, desde el inicio se registró una baja transparencia del agua. Esto puede deberse a que su principal afluente es el río Turbio, el cual lleva las aguas negras de León, una ciudad industrializada. La transparencia del agua disminuyó poco a lo largo del estudio y la diversidad de zooplancton y macroinvertebrados no tuvo un cambio significativo.

Se detectaron altos valores de SDT tanto en Coyote como en Silva, estos son más altos que los recomendados para proteger la vida acuática de acuerdo con la norma oficial mexicana NOM-001-SEMARNAT-1996, que establece un límite de TDS de 60 mg / L (http://diariooficial. gob.mx/nota_detalle.php?codigo=4863829\&fecha=06/01/1997). Estudios demuestran que patrones muy altos de SDT también pueden disminuir la abundancia del zooplancton (Ivanova y Kazantseva, 2006).

Además de los procesos dentro del ecosistema acuático mencionados anteriormente, las actividades humanas externas también pueden empeorar la eutrofización de los humedales (Kuczynska-Kippen y Joniak, 2016).

\begin{tabular}{|c|c|c|c|c|c|}
\hline 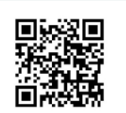 & (c) (1) $\$$ & $\underbrace{}_{\text {AMBIFNAIIES }}$ & $\begin{array}{l}\frac{9 \%}{2} \\
\frac{2}{2} \\
\text { euna }\end{array}$ & 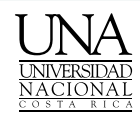 & 150 \\
\hline
\end{tabular}




\section{Revista de CIENCIAS AMBIENTALES Tropical Journal of Environmental Sciences}

Revista de Ciencias Ambientales (Trop J Environ Sci) e-ISSN: 2215-3896

(Julio-Diciembre, 2021) . Vol 55(2): 141-156 DOI: https://doi.org/10.15359/rca.55-2.7

Open Access: www.revistas.una.ac.cr/ambientales e-mail: revista.ambientales@una.ac.cr Araiza-Ortiz M., Zambrano-González L., Mazari-Hiriart M. y Suzán G.

Los valores de $\delta^{15} \mathrm{~N}$ y $\delta^{13} \mathrm{C}$ de los organismos acuáticos muestreados en Silva y Coyote reflejaron la introducción de nitrógeno y carbono proveniente de las actividades humanas. Los valores de $\delta^{15} \mathrm{~N}$ coincidieron con los valores usualmente encontrados en aguas residuales, de $10 \%$ a $20 \%$ (McClelland y Valiela, 1998), así como de fertilizantes utilizados para la agricultura, de $15 \%$ a $30 \%$ (Cole et al., 2004). Los valores de $\delta^{13} \mathrm{C}$ también coincidieron con los valores encontrados en los granos comúnmente sembrados en la zona, como maíz y trigo, de -9 \%o a -17 \%o (Hamilton et al., 1992).

La eutrofización encontrada en Silva y Coyote, junto con temperaturas superiores a $20^{\circ} \mathrm{C}$, comunes en la región aún en otoño e invierno, pueden proporcionar las condiciones idóneas para que se reproduzca la bacteria del botulismo y por lo tanto, se libere la toxina (Babinszky et al., 2008). Durante la realización de este estudio se presentó un brote de mortalidad de aves acuáticas. La confirmación de presencia de la toxina botulínica en la sangre de los patos muertos, mediante la prueba de bioensayo en ratón confirmó que se trataba de un brote de botulismo aviar.

En otros humedales someros tropicales donde la eutrofización y las altas temperaturas han ocasionado brotes de botulismo aviar, se ha logrado controlar el brote aumentando el nivel del agua; esto disminuye la temperatura, además de la carga de nitrógeno y fósforo, por lo que ya no hay condiciones ideales para la reproducción de la toxina (Brandis et al., 2020; Son et al., 2018). Esta estrategia no es posible en los presentes sitios de estudio, ya que no existe una fuente de agua permanente cerca.

Aunque en Refugio de Trancas el nivel del agua también disminuyó a lo largo del periodo de estudio, no presentó una sequía completa. Al no haber materia orgánica en descomposición, ni zonas anóxicas o temperaturas mayores a $20^{\circ} \mathrm{C}$, no existe un ambiente propicio para la liberación de la toxina botulínica. Los valores de los isótopos estables de los organismos muestreados tampoco reflejaron el influjo de nutrientes provenientes de las actividades humanas. Este humedal es el que tiene un menor porcentaje de agricultura a su alrededor.

Con el cambio climático, el impacto del botulismo aviar en los humedales someros tropicales puede incrementarse cada vez más y tener consecuencias a largo plazo para las especies de aves amenazadas que ahí habitan (Russell et al., 2019). Las altas temperaturas, junto los cambios en la precipitación en zonas sin afluentes permanentes, además de la alta carga de nutrientes, hace más propensos a este tipo de humedales a la eutrofización (Menezes, 2019). En el caso de las aves acuáticas migratorias puede ser aún más perjudicial, ya que los brotes de botulismo no solo podrían ocurrir a finales de verano y otoño en sus sitios de reunión antes de migrar, si no también en sus sitios de invernación.

Por lo tanto, es necesario establecer medidas de restauración dirigidas a reducir la carga de nutrientes y de materia orgánica en descomposición, tales como: aumentar el nivel del agua en aquellos lugares donde sea posible, la eliminación de vegetación terrestre antes de la temporada de lluvias en los humedales no permanentes, así como la exclusión de los peces no nativos.

Esta última medida ha dado buenos resultados en los programas de restauración de los lagos someros (Scheffer y Ness, 2007). Los peces del género Oreochromis, además de aportar más

\begin{tabular}{|c|c|c|}
\hline 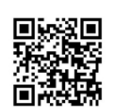 & (c) $\underset{\mathrm{BY}}{\mathrm{NC}} \mathrm{SA}_{\mathrm{SA}}$ & 151 \\
\hline
\end{tabular}




\section{Revista de CIENCIAS AMBIENTALES Tropical Journal of Environmental Sciences}

Revista de Ciencias Ambientales (Trop J Environ Sci) e-ISSN: 2215-3896

(Julio-Diciembre, 2021) . Vol 55(2): 141-156 DOI: https://doi.org/10.15359/rca.55-2.7

Open Access: www.revistas.una.ac.cr/ambientales e-mail: revista.ambientales@una.ac.cr Araiza-Ortiz M., Zambrano-González L., Mazari-Hiriart M. y Suzán G.

nutrientes al sistema y favorecer a la resuspensión del sedimento, se han asociado de manera directa a la presentación de brotes de botulismo aviar, ya que se ha detectado la presencia de la toxina botulínica en sus heces (Nol et al., 2004).

Otras acciones podrían ser el uso de productos químicos para reducir la materia orgánica y las partículas suspendidas (Araújo et al., 2015). Se recomienda un programa piloto de restauración basado en el establecimiento de mesocosmos, utilizando diferentes tratamientos para identificar cuales acciones de manejo serían adecuadas para cada humedal.

\section{Conclusiones}

Hubo cambios, tanto bióticos como fisicoquímicos, a lo largo del año en los tres humedales estudiados. La diversidad de zooplancton y macroinvertebrados disminuyó en todos los sitios. En los humedales no permanentes, como Silva y Coyote, en donde se han presentado brotes recurrentes de botulismo, la inundación en época de lluvias después de una sequía completa ocasionó la muerte de la vegetación terrestre, proporcionando una gran cantidad de materia orgánica en descomposición.

La introducción de peces y la llegada de miles de aves migratorias añadieron más nutrientes al sistema, esto se reflejó en una menor transparencia del agua, niveles altos de solidos disueltos totales y zonas anóxicas. Asimismo, se comprobó el flujo de nutrientes provenientes de las actividades humanas como la agricultura y el flujo de aguas residuales hacia estos humedales. Estas condiciones eutróficas, junto con las temperaturas elevadas encontradas, podrían ocasionar brotes recurrentes de botulismo aviar.

En el humedal permanente Refugio de Trancas, donde no se han registrado brotes, la transparencia disminuyó poco a lo largo del estudio. No se detectó flujo de nutrientes causados por actividades humanas hacia el humedal. Las características ambientales observadas como temperaturas más bajas y zonas no anóxicas no son apropiadas para que se libere la toxina botulínica.

Con el cambio climático, el impacto del botulismo aviar en los humedales someros tropicales semi-aridos puede incrementarse cada vez más y tener consecuencias a largo plazo para las aves acuáticas. Por lo tanto, es necesario establecer medidas de restauración dirigidas a reducir la carga de nutrientes. Se recomienda un programa piloto de restauración basado en el establecimiento de mesocosmos, utilizando diferentes tratamientos para identificar cuáles acciones de manejo serían adecuadas para cada humedal.

\section{6. Ética y conflicto de intereses}

Las personas autoras declaramos que hemos cumplido totalmente con todos los requisitos éticos y legales pertinentes, tanto durante el estudio como en la producción del manuscrito; no hay conflictos de intereses de ningún tipo; todas las fuentes financieras se mencionan completa y claramente en la sección de agradecimientos; y estamos totalmente de acuerdo con la versión final editada del artículo.

\begin{tabular}{|c|c|c|c|c|}
\hline 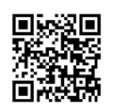 & (c) (1) & $\overbrace{\text { AMBENIIIIS }}$ & $\frac{\mid \%}{\text { euna }}$ & 152 \\
\hline
\end{tabular}




\section{Revista de CIENCIAS AMBIENTALES Tropical Journal of Environmental Sciences}

Revista de Ciencias Ambientales (Trop J Environ Sci) e-ISSN: 2215-3896

(Julio-Diciembre, 2021) . Vol 55(2): 141-156 DOI: https://doi.org/10.15359/rca.55-2.7

Open Access: www.revistas.una.ac.cr/ambientales e-mail: revista.ambientales@una.ac.cr Araiza-Ortiz M., Zambrano-González L., Mazari-Hiriart M. y Suzán G.

\section{Agradecimientos}

Agradecemos a International Wild Waterfowl Association (IWWA) por el financiamiento para realizar el estudio de isotopos estables, a la Procuraduría Ambiental y de Ordenamiento Territorial del Estado de Guanajuato (PAOT), a la Secretaría del Medio Ambiente y Ordenamiento Territorial del Estado de Guanajuato (SMAOT) y al Instituto Tecnológico de Estudios Superiores de Irapuato (ITESI) por el apoyo con el material para el muestreo y el uso de sus laboratorios. También al municipio de San Francisco del Rincón, E. Corona, D Rogelio y S. Arguelles $(\dagger)$ por su ayuda en la captura de los patos. Marcela Araiza agradece a la Dirección General de Estudios de Posgrado, Posgrado en Ciencias Biológicas, UNAM por su apoyo con la beca de CONACYT No. 384970/261085. Finalmente, agradecemos a la Revista y las personas anónimas, con cuya contribución se mejoró el artículo final.

\section{Referencias}

Araújo, F., Becker, V., \& Attayde, J. L. (2015). Shallow lake restoration and water quality management by the combined effects of polyaluminium chloride addition and benthivores fish removal: a field mesocosm experiment. Hydrobiologia, 778(1), 243-252. https://doi.org/10.1007/ s10750-015-2606-5

Babinszky, G., Csitári, G., \& Józsa, S. (2008). Observations on environmental factors in connection with avian botulism outbreaks in a Hungarian wetland habitat. Acta Microbiologica et Immunologica Hungarica, 55(4), 455-464. https://doi.org/10.1556/AMicr.55.2008.4.10

Brandis, K. J., Spencer, J., Wolfenden, B., Palmer, D. (2020). Avian-botulism risk in waterbird breeding colonies and implications for environmental water management. Marine and Freshwater Research, 71(2), 179-190. https://doi.org/10.1071/MF18446

CEC (1995). Secretariat report of the death of migratory birds at the Silva Reservoir (1994-1995). Report of the Commission for Environmental Cooperation Secretariat. Oaxaca, Mexico.

Cole, M. L., Valiela, I., Kroeger, K. D., Tomasky, G. L., Cebrain, J., Wigand, C., McKinney, R. A., Grady, S. P., Carvalho da Silva, M. H. (2004). Assessment of a $\delta$ N Isotopic Method to Indicate Anthropogenic Eutrophication in Aquatic Ecosystems. Journal of Environmental Quality, 33(1),124-132. https://doi.org/10.2134/jeq2004.1240

Comín, F.A. y Herrera, J.A. (2000). The role of birds on the trophic structure and nutrient cycles of aquatic ecosystems. En F.A. Comin, J.A. Herrera. (Eds.) Proceedings of an international symposium of Limnology and aquatic birds, monitoring, modelling and management (pp. 205208). Universidad Autónoma de Yucatán.

Craig, H. (1957). Isotopic standards for carbon and oxygen and correction factors for mass spectrometric analysis of carbon dioxide. Geochimica et Cosmochimica Acta, 12, 133-149. https:// doi.org/10.1016/0016-7037(57)90024-8

\begin{tabular}{|c|c|c|}
\hline 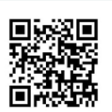 & (c) (i) () () & 153 \\
\hline
\end{tabular}




\section{Revista de CIENCIAS AMBIENTALES Tropical Journal of Environmental Sciences}

Revista de Ciencias Ambientales (Trop J Environ Sci) e-ISSN: 2215-3896

(Julio-Diciembre, 2021) . Vol 55(2): 141-156 DOI: https://doi.org/10.15359/rca.55-2.7

Open Access: www.revistas.una.ac.cr/ambientales e-mail: revista.ambientales@una.ac.cr Araiza-Ortiz M., Zambrano-González L., Mazari-Hiriart M. y Suzán G.

Domínguez, M.A. (2009). Análisis prospectivo de los factores que intervienen en la presentación de brotes de botulismo en aves acuáticas [Tesis de licenciatura]. Universidad Nacional Autónoma de México, México.

Evans, A.E., Mateo-Sagasta, J., Qadir, M., Boelee, E., Ippolito, A. (2019). Agricultural water pollution: key knowledge gaps and research needs. Current Opinion in Environmental Sustainability, 36, 20-27. https://doi.org/10.1016/j.cosust.2018.10.003

Hamilton, S.K., Lewis, W.M., Sippel, S.J. (1992). Energy sources for aquatic animals in the Orinoco River floodplain: evidence from stable isotopes. Oecologia, 89(3), 324-330. https://doi. org/10.1007/BF00317409

Havens, K., Paerl, H., Phlips, E., Zhu, M., Beaver, J., Srifa, A. (2016). Extreme weather events and climate variability provide a lens to how shallow lakes may respond to climate change. Water, 8(6), 229. https://doi.org/10.3390/w8060229

Hebert, C. E., Wassenaar, L.I. (2001). Stable nitrogen isotopes in waterfowl feathers reflect agricultural land use in western Canada. Environmental science \& technology, 35(17), 3482-3487. https://doi.org/10.1021/es001970p

Ivanova, M.B., Kazantseva, T. I. (2006). Effect of water $\mathrm{pH}$ and total dissolved solids on the species diversity of pelagic zooplankton in lakes: A statistical analysis. Russian Journal of Ecology, 37(4), 264-270. https://doi.org/10.1134/S1067413606040084

Jardine, T. D., McGeachy, S. A., Paton, C. M., Savoie, M., Cunjak, R. A. (2003). Stable isotopes in aquatic systems: simple preparation, analysis and interpetation. Canadian manuscript report of fisheries and aquatic sciences Halieut Aquat, 2656, 44.

Kuczynska-Kippen, N., Joniak, T. (2016). Zooplankton diversity and macrophyte biometry in shallow water bodies of various trophic state. Hydrobiologia, 774(1), 39-51. https://doi. org/10.1007/s10750-015-2595-4

McClelland, J. W., Valiela, I. (1998). Linking nitrogen in estuarine producers to and-derived sources. Limnology and Oceanography, 43(4), 577-585. https://doi.org/10.4319/lo.1998.43.4.0577

Menezes, R. F., Attayde, J. L., Kosten, S., Lacerot, G., e Souza, L. C., Costa, L. S., Jeppesen, E. (2019). Differences in food webs and trophic states of Brazilian tropical humid and semi-arid shallow lakes: implications of climate change. Hydrobiologia, 829(1), 95-111. https://doi. org/10.1007/s10750-018-3626-8

Michener, R., Lajtha, K. (2007). Stable Isotopes in Ecology and Environmental Science. Blackwell Publishing, NY. https://doi.org/10.1002/9780470691854

\begin{tabular}{|c|c|c|c|c|}
\hline 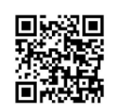 & (c) () & $\underset{\text { AMBENENALIES }}{\infty}$ & $\frac{1 \%}{2 \%}$ & 154 \\
\hline
\end{tabular}




\section{Revista de CIENCIAS AMBIENTALES Tropical Journal of Environmental Sciences}

Revista de Ciencias Ambientales (Trop J Environ Sci) e-ISSN: 2215-3896

(Julio-Diciembre, 2021) . Vol 55(2): 141-156 DOI: https://doi.org/10.15359/rca.55-2.7

Open Access: www.revistas.una.ac.cr/ambientales e-mail: revista.ambientales@una.ac.cr Araiza-Ortiz M., Zambrano-González L., Mazari-Hiriart M. y Suzán G.

Murakani, M., Harada, S., Ichiyanagi, H., Susuki, T., Yamagishi, S. (2015). Water reservoirs of non-breeding waterfowl: the importance of shallow areas for maintaining diversity. Bird Study, 62(3), 417-422. https://doi.org/10.1080/00063657.2015.1059798

Murphy, T., Lawson, A., Nalewajko, C., Murkin, H., Ross, L., Oguma, K., Mclntre, T. (2000). Algal toxins-initiators of avian botulism? Environmental Toxicology, 15(5), 558-567. https:// doi.org/10.1002/1522-7278(2000)15:5<558::AID-TOX29>3.0.CO,2-R

Nol, P., Rocke, T.E., Gross, K., Yuill, T.M. (2004). Prevalence of neurotoxic Clostridium botulinum type $\mathrm{C}$ in the gastrointestinal tracts of tilapia (Oreochromis mossambicus) in the Salton Sea. Journal of Wildlife Disease, 40(3), 414-419. https://doi.org/10.7589/0090-3558-40.3.414

Ortiz, N.E., Smith, G.R. (1994). Landfill sites, botulism and gulls. Epidemiology and Infection, 112(2), 385-391. https://doi.org/10.1017/S0950268800057794

Rocke, T. E., Bollinger, T. K. (2007). Avian botulism. En Thomas, N. J., y D. B. Hunter. Infectious diseases of wild birds (pp. 377-416). Blackwell Publishing. https://doi. org/10.1002/9780470344668.ch21

Russell, I. A., Randall, R. M., Zimmerman, D., Govender, D. (2019). Outbreak of avian botulism and its effect on waterbirds in the wilderness lakes, South Africa. Koedoe, 61, a1553. https:// doi.org/10.4102/koedoe.v61i1.1553

Scheffer, M., van Ness, E.H. (2007). Shallow lakes theory revisted: various alternative regimes by climate, nutrients, depth and lake size. Hydrobiologia, 584(1), 455-466. https://doi. org/10.1007/978-1-4020-6399-2_41

Son, K., Kim, Y. K., Woo, C., Wang, S. J., Kim, Y., Oem, J. K., Jheong, W., Jeong, J. (2018). Minimizing an outbreak of avian botulism (Clostridium botulinum type C) in Incheon, South Korea. Journal of Veterinary Medical Science, 17-0519. https://doi.org/10.1292/jvms.17-0519

Thornton, S.F., McManus, J. (1994). Application of organic carbon and nitrogen stable isotope and $\mathrm{C} / \mathrm{N}$ ratios as source indicators of organic matter provenance in estuarine systems: Evidence from the Tay Estuary, Scotland. Estuarine Coastal and Shelf Science, 38(3), 219-233. https://doi.org/10.1006/ecss.1994.1015

Usio, N., Nakagawa, M., Aoki, T., Higuchi, S., Kadono, Y., Akasaka, M., Takamura, N. (2017). Effects of land use on trophic states and multi-taxonomic diversity in Japanese farm ponds. Agriculture Ecosystems and Environment, 247, 205-2015. https://doi.org/10.1016/j. agee.2017.06.043

van Altena, C., Bakker, E.S., Kuiper, J. J., Mooij, W. M. (2016). The impact of bird herbivory on macrophytes and the resilience of the clear-water state in shallow lakes: a model study. $\mathrm{Hy}$ drobiologia, 777(1), 197-207. https://doi.org/10.1007/s10750-016-2779-6

\begin{tabular}{|c|c|c|}
\hline 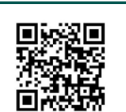 & (c) $\underset{\mathrm{BY}}{(i)} \mathrm{Na}_{\mathrm{SA}}$ & 155 \\
\hline
\end{tabular}




\section{Revista de CIENCIAS AMBIENTALES

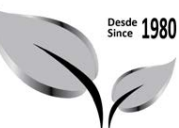 Tropical Journal of Environmental Sciences}

Revista de Ciencias Ambientales (Trop J Environ Sci) e-ISSN: 2215-3896

(Julio-Diciembre, 2021) . Vol 55(2): 141-156 DOI: https://doi.org/10.15359/rca.55-2.7 Open Access: www.revistas.una.ac.cr/ambientales e-mail: revista.ambientales@una.ac.cr Araiza-Ortiz M., Zambrano-González L., Mazari-Hiriart M. y Suzán G.

Wray, A. K., Bell, D. A., Dramer, P., Taylor, M. (2016). Waterbird susceptibility to avian cholera at Haywars Marsh, California, USA. Journal of Wildlife Diseases, 52(3), 699-704. https://doi. org/10.7589/2015-11-306 